\title{
EH 抗燃油劣化原因及其净化与再生研究
}

\author{
徐 康 \\ 国家能源集团棟壁发电厂，江苏 镇江 212006
}

[摘要] 抗燃油在火电厂发电生产中有着重要应用，但从抗燃油实际使用情况看，存在抗燃油使用后出现劣化的问题，导致发 电设备运行出现故障。文中主要针对 EH 抗燃油劣化原因及再生研究等方面展开讨论，结合抗燃油使用具体情况，提出改善劣 化问题的措施，改进油品处理方式，最终达到机组安全生产的效果。

[关键词] EH 抗燃油; 劣化原因; 净化与再生

DOI: $10.33142 /$ ec.v2i9.694

中图分类号: TM621

文献标识码：A

\section{Study on the Causes of EH Anti-fuel Degradation and its Purification and Regeneration XU Kang \\ Jianbi Power Plant of National Energy Group, Jiangsu Zhenjiang, 212006 China}

\begin{abstract}
Anti-fuel has an important application in power generation production of thermal power plant, but from the actual use of anti-fuel, there is a problem of deterioration after the use of anti-fuel, which leads to the operation of power generation equipment. In this paper, the causes of anti-fuel deterioration and regeneration of $\mathrm{EH}$ are discussed, and combined with the specific situation of anti-fuel use, the measures to improve the deterioration problem are put forward, and the treatment method of oil products is improved, so as to achieve the effect of unit safety production.
\end{abstract}

Keywords: EH anti-fuel oil; Cause of degradation; Purification and regeneration

\section{引言}

大型汽轮机作业过程中, 要充分发挥调速系统作用, 其中抗燃油是调速系统中液压介质的一种。在油品使用过程 中, 容易受到外界水分、空气和作业环境等因素的影响发生劣化, 并且设备操作不当将加速抗燃油的变质。因此, 采 用正确的方法和合适的设备尽可能防范抗燃油出现劣化以及出现劣化后如何净化再生就显得尤为重要。

\section{EH 抗燃油劣化原因}

抗燃油属于酯类物质范畴, 实际使用中容易出现油品劣化现象, 由此引起调速系统故障导致发电机组跳机, 当前 防范抗燃油劣化已成为该领域主要研究问题, 进行劣化油品的净化和再生处理, 不仅能提高抗燃油应用效益, 还能提 高设备运作安全性。下面本文将具体分析抗燃油劣化的原因，以便采取有效的防范措施。

\section{1 非金属物质对抗燃油性质的影响}

抗燃油对非金属物质较敏感, 主要是由于它的溶剂效应, 能溶解较多非金属材料, 包括密封祄垫、皮囊破损物等, 这些材料融入抗燃油中势必会影响油品性质, 加速油品劣化, 由此不能发挥抗燃油功能, 对机组运行状况产生直接影 响。常用的天然橡胶、丁腈橡胶等密封材料, 与磷酸酯抗燃油的相容性较差, 在设备运行中, 橡胶材料将进入抗燃油 中。在这种环境下, 检查抗燃油时通常会发现其中存在黑色沉淀物, 检测可知其为蓄能器破损物。一般来讲, 抗燃油 对于一些聚合材料和有机化合物体现出较强溶解能力, 对普通耐油橡胶材料有着溶胀效果。因此, 选择设备的密封材 料时, 要重点考虑材料和抗燃油的适应性问题。根据已有经验选择适当的非金属材料, 并在试验达标后将其运用到机 组系统设计中。

\section{2 温度的影响}

磷酸酯抗燃油属于人工合成油, 相对来讲化学稳定性差, 当受到水分、高温、杂质影响时, 将破坏抗燃油性能, 尤其在高温作业环境下, 将造成油品分解。尽管抗燃油能起到抗燃的作用, 但对环境温度有较高要求。常温情况下, 抗燃油氧化速度缓慢, 随着温度升高将加速其氧化速度, 由此可知, 要想保证抗燃油正常使用, 要加大对环境温度的 控制。实践表明，抗燃油使用环境温度应保持在 $40-55^{\circ} \mathrm{C}$ 之间，避免长期在高温下使用。温度较高时，还会对抗燃油酸 值带来影响, 如果在高温条件下使用抗燃油, 则油品发生氧化和裂解反应, 由此带来密封材料被溶解和酸值增加的后 果, 不仅降低了抗燃油性质, 还会导致系统密封性变差。尤其油动机附近温度较高, 是需要进行温度控制的重要部位。

\section{3 水分的影响}

抗燃油使用过程中，可能会受到水分影响，这些水分来源于油箱顶部呼吸器吸入空气中的水分，以及抗燃油老化 
产生的水分。 $\mathrm{EH}$ 抗燃油接触水分后, 将生成羧酸和酚, 其中羧酸起到催化作用, 由此加速了油品劣化, 给整个机组运 行带来安全隐患。上述反应的发生, 还会造成抗燃油酸值增加, 将会对密封材料和内部构件造成腐蚀, 导致油液渗漏。 在使用抗燃油时, 要加大对 $\mathrm{EH}$ 油温度的控制, 同时应尽可能减少空气流入呼吸器中结成水珠, 从上述角度出发实施防 范策略, 能有效抑制抗燃油的劣化。

\section{2 劣化现象防范和再生措施}

从以上阐述可知, 抗燃油使用过程中主要受到水分、环境温度和非金属材料的影响, 导致 $\mathrm{EH}$ 抗燃油劣化, 因此, 要从上述角度出发, 减少上述因素对油品性质的影响。如果抗燃油发生劣化, 必须采取合适措施进行油质再生, 以确 保抗燃油安全可靠使用，保证发电机组不发生因抗燃油劣化导致的故障。

\section{1 加强机组运行期间的维护工作}

在进行机组日常维护工作时, 树立防范抗油剂劣化的维护意识, 有目的的进行维护作业。首先, 需要保证滤油工 作正常开展, 每台机组每周运行一次抗燃油再生系统。目前我厂已弃用硅藻土再生方式, 使用的再生滤芯为 PALL 公司 生产的阴、阳离子再生滤芯, 阴离子降低酸值, 阳离子提高电阻率, 每次每种滤芯各运行 6 小时。再生过程中由于离 子交换会产生较多水分, 因此必须同时投用脱水滤油机, 脱水滤油机与再生装置投用时间比不能低于 $3: 1$, 以保证水分 $<1000 \mathrm{mg} / \mathrm{L}$ 。其次, 在机组运行管理中, 要进行针对油温、抗燃油油位、流量及泵压力的控制, 将系统各环节的油压测 定作为日常检查项目, 及时调整系统运行不当的问题。最后, 需要每季度至少进行一次油质全分析, 主要检查项目包 括密度、电阻率、外观、颜色、酸值、运动粘度、矿物油含量、泡沫特性等。当抗燃油品质出现异常状况时, 要进行 原因分析，及早发现问题。

\section{2 加大对抗燃油重要指标的监控}

发电厂自己的油化验人员应每月自行检查酸值、电阻率、颗粒度和水分这四个重要指标, 并做好记录, 加强油质 变化趋势的监控。一是酸值指标, 高酸值将引起油品沉淀、空气间隔及气泡等问题。当油品酸度 $>0.15 \mathrm{mgKOH} / \mathrm{g}$ 时, 需 要增加阴离子再生滤芯的投用时间, 同时相应增加脱水时间, 避免离子交换导致油中含有大量水分。二是含水量控制, 随着抗燃油的水解, 将产生酸性产物, 会造成敏感部件被侵蚀。因此, 要将含水量控制作为重点管理内容。目前效果 较好的脱水方式是使用真空脱水滤油机, 但真空脱水滤油机也不宜长期连续投用, 否则会加速油质老化速度, 应根据 油质报告和再生装置投用时间决定真空脱水滤油机的投用时间。三是电阻率指标，电阻率是水分、酸值、金属离子以 及其他因素综合作用的结果, 如果偏低会引起电液伺服阀的液压元件发生电化学腐蚀, 所以发现电阻率 $>5 \times 10^{9} \Omega \cdot \mathrm{cm}$ 后必须及时投用阳离子再生滤芯进行滤油。四是颗粒度, 如果>6 级 (NAS1638 标准), 表明抗燃油已被污染, 必须立即 进行除杂质过滤, 滤芯精度不得低于 $3 \mu \mathrm{m}$ 。

\section{3 发生劣化后的净化和再生措施建议}

以我厂某一机组为例, 在运行中发现抗燃油出现劣化现象, 酸值超标达 $0.289 \mathrm{mgKOH} / \mathrm{g}$, 立即联系运行投用 PALL 公司产 PFS8370R3CH81 型再生装置, 并将通路切换为阴离子再生滤芯，每天投用 8h，连续投用 4 天。同时规定 24 小时 不停歇投用 PALL 公司产 HNP021-X109 型移动式净油机配合脱水 4 天。该净油机能够通过真空调节清除抗燃油中 $100 \%$ 游离水和高达 $90 \%$ 的溶解水、100\%的游离和混入的气体以及 $80 \%$ 的溶解气体, 当污油经过喷嘴进入到净油机真空容器的 顶部, 由于重力作用下降到容器底部, 而空气和水被气流从容器顶部除去 (具体原理此处不作详述)。同时净油机通过 出口拦截滤芯能够有效清除淤泥和其他固体或颗粒污物, 从而达到脱水降颗粒度一举两得的功效。经过 4 天滤油, 再 次取样化验指标, 酸值已达标, 继续巩固滤油 3 天后通知运行恢复常规维护滤油频率。电阻率超标也可按此方法再生 过滤, 而水分或颗粒度超标只需连续 4 天单独投用 HNP021-X109 型移动式净油机即可。若按此方法连续过滤一个月指 标仍未合格，表明油品已重度污染无法再生，须考虑更换新抗燃油。

\section{结论}

综上所述, 抗燃油劣化将对机电设备运行效益和质量产生不良影响, 引起劣化问题的原因较多, 要在明确抗燃油 劣化原因的基础上, 实施可行性防范措施。一旦发生劣化, 必须按照正确方法及时投用合适的再生装置, 以便纠正油 品劣化问题, 延长抗燃油的使用寿命。

\section{[参考文献]}

[1]吕修业.磷酸酯抗燃油劣化的原因分析及处理 [J].山东工业技术,2018(24): 55-56.

[2] 郭华,钱玉良。磷酸酯抗燃油酸值、电阻率指标劣化原因分析及控制措施 [J].浙江冶金, 2017 (01) : 42-43.

作者简介: 徐康, 男，(1982.11-）,工程师，从事火电厂汽轮机系统检修专业。 